01

\title{
Изотропные матричные элементы интеграла столкновений уравнения Больцмана
}

\author{
( С И.А. Эндер, ${ }^{1}$ Л.А. Бакалейников, ${ }^{1}$ Е.Ю. Флегонтова, ${ }^{1,}$ Ф А.Б. Герасименко ${ }^{1}$ \\ ${ }^{1}$ Физико-технический институт им. А.Ф. Иофрфе РАН, \\ 194021 Санкт-Петербург, Россия \\ ${ }^{2}$ Санкт-Петербургский государственный университет, \\ 194021 Санкт-Петербург, Россия \\ ^e-mail: fl.xiees@mail.ioffe.ru
}

(Поступило в Редакцию 6 февраля 2017 г.)

Предложен алгоритм построения матричных элементов интеграла столкновений изотропного по скоростям нелинейного уравнения Больцмана. Эти матричные элементы используются в качестве стартовых в рекуррентной процедуре расчета матричных элементов неизотропного по скоростям интеграла столкновений, изложенной в нашей предыдущей работе. Кроме того, изотропные матричные элементы представляют и самостоятельный интерес для расчета изотропной релаксации в ряде задач физико-химической кинетики. Показано, что коэффициенты разложения изотропных матричных элементов по $\Omega$-интегралам связаны рекуррентными соотношениями, позволяющими построить процедуру их последовательного определения.

DOI: 10.21883/JTF.2017.09.44900.2192

\section{Введение}

Развитие современной техники как за счет миниатюризации устройств, так и за счет перехода к более напряженным режимам работы требует решения кинетических задач на уровне функции распределения (ФР). Информация о ФР в области больших скоростей оказывается определяющей и для расчета физико-химических процессов в газе и плазме. Наконец, к необходимости разработки методов расчета ФР в сильно неравновесной ситуации приводит и естественное развитие самой кинетической теории. Одним из методов решения уравнения Больцмана при сильном отклонении от равновесия является метод полиномиальных разложений, в частности моментный метод, в котором ФР раскладывается по функциям Барнетта $H_{r, l, m}=S_{l+1 / 2}^{r}(x) Y_{l, m}(\theta, \phi)$, где $S_{l+1 / 2}^{r}(x)$ - полиномы Сонина, $Y_{l, m}(\theta, \phi)$ - сферические гармоники.

Основной причиной, сдерживающей развитие этого метода, было отсутствие достаточного количества матричных элементов интеграла столкновений (МЭ). В кинетической теории газов, как правило, вычислялись неизотропные МЭ с малыми индексами $l[1,2]$. Это связано с их исключительной ролью при вычислении коэффициентов переноса при малых отклонениях от равновесия. В сильно неравновесных процессах для аккуратного построения ФР необходимо знание МЭ с большими индексами. Существующие прямые аналитические формулы для определения МЭ (см., например, [3]) чрезвычайно громоздки. В [4,5] был предложен значительно более простой способ последовательного определения МЭ с помощью рекуррентных процедур.
Настоящая работа тесно связана с нашей предыдущей работой [6], в которой описана такая рекурретная процедура построения любых неизотропных (соответствующих неизотропной по скоростям ФР) матричных элементов. При этом в качестве стартовых предлагается использовать изотропные МЭ. Важно отметить, что сведения о сечениях взаимодействия используются только на этапе построения изотропных МЭ.

В работе будет показано, что любые изотропные МЭ, как линейные, так и нелинейные можно представить в виде линейной комбинации $\Omega$-интегралов, коэффициенты которых связаны алгебраическими рекуррентными соотношениями.

Следует отметить, что построение изотропных МЭ важно не только для завершения нашей рекуррентной процедуры. Сравнительно недавно появилась работа Шизгала и Дриди [7], в которой вычисляются коэффициенты разложения линейных изотропных МЭ по $\Omega$ интегралам. В этой работе описывается целая серия прикладных задач, для которых важно знание линейных изотропных МЭ. Это важно, например, при расчете ФР небольшой примеси атомов (или ионов) на фоне равновесно распределенных атомов другого сорта.

В [7] подчеркивается роль изотропной релаксации, т. е. этапа, на котором ФР релаксирует, оставаясь изотропной. Скорости физико-химических превращений на этом этапе определяются поведением ФР в области больших скоростей.

Итак, разложение изотропных матричных элементов по $\Omega$ - интегралам важно как с точки зрения получения стартовых МЭ для применения рекуррентных процедур, так и для решения ряда релаксационных задач, необходимых, в частности, для физико-химической кинетики. 


\section{Постановка задачи}

Нелинейные интегралы столкновений для каждой пары компонентов газовой смеси с массами $m_{a}$ и $m_{b}$ и плотностями $n_{a}$ и $n_{b}$ в случае изотропных по скоростям $\Phi \mathrm{P}\left(f_{a}(\mathbf{v})=f_{a}(v)\right)$ имеют вид

$$
\begin{aligned}
\hat{I}\left(f_{a}, f_{b}\right)= & n_{a} n_{b} \int\left(f_{a}\left(v_{1}\right) f_{b}\left(v_{2}\right)\right. \\
& \left.-f_{a}(v) f_{b}\left(v^{\prime}\right)\right) g \sigma_{a b}(g, \theta) d \mathbf{v}^{\prime} d \mathbf{k} .
\end{aligned}
$$

Скорости частиц до и после столкновения связаны соотношениями

$$
\begin{gathered}
\mathbf{v}_{1}=\mathbf{v}_{0}-\mu_{b} \mathbf{k} g, \mathbf{v}_{2}=\mathbf{v}_{0}+\mu_{a} \mathbf{k} g, \mathbf{v}_{0}=\left(\mu_{a} \mathbf{v}+\mu_{b} \mathbf{v}^{\prime}\right), \\
\mu_{a}=m_{a} /\left(m_{a}+m_{b}\right), \mu_{b}=m_{b} /\left(m_{a}+m_{b}\right), \\
\mathbf{g}=\mathbf{v}_{1}-\mathbf{v}_{2}, \mathbf{g}^{\prime}=\mathbf{v}-\mathbf{v}^{\prime}, g=g^{\prime},
\end{gathered}
$$

где $\mathbf{k}$ - единичный вектор, направленный вдоль $\mathbf{g}$. Угол рассеяния $\theta$ определяется соотношением $\cos \theta=$ $=\mathbf{k} \cdot \mathbf{g}^{\prime} / g$, а $\sigma_{a b}(g, \theta)$ - дифференциальное сечение рссеяния.

При применении моментного метода к изотропным по скоростям задачам ФР $a$-й компоненты смеси $f_{a}(v)$ разлагают по полиномам Сонина $S_{1 / 2}^{r}(x)$ с максвелловским весом $M_{a}$

$$
\begin{gathered}
f_{a}(v, t)=M_{a} \sum_{r=0}^{\infty} C_{r}^{a}(t) S_{1 / 2}^{r}\left(\frac{m_{a} v^{2}}{2 k T}\right), \\
M_{a}=\left(\frac{m_{a}}{2 k T \pi}\right)^{3 / 2} \exp \left(\frac{-m_{a} v^{2}}{2 k T}\right) .
\end{gathered}
$$

Здесь $k-$ постоянная Больцмана, $T-$ температура весового максвеллиана. Изотропные матричные элементы $K_{r_{1}, r_{2}}^{r}$ определяются через интегралы столкновений $\hat{I}\left(f_{a}, f_{b}\right)$ следующим образом:

$$
\begin{aligned}
& \left(K_{a, b}^{a}\right)_{r_{1}, r_{2}}^{r}=\frac{4 \pi}{v_{r}} \\
& \times\left(\int_{0}^{\infty} S_{1 / 2}^{r}\left(c_{a}^{2}\right) \hat{I}_{a, b}\left(M_{a} S_{1 / 2}^{r_{1}}\left(c_{a}^{2}\right), M_{b} S_{1 / 2}^{r_{2}}\left(c_{b}^{2}\right)\right) v^{2} d v\right), \\
& c_{a}=\left(\frac{m_{a}}{2 k T}\right)^{1 / 2} v, c_{b}=\left(\frac{m_{b}}{2 k T}\right)^{1 / 2} v, v_{r}=\frac{(2 r+1) ! !}{(2 r) ! !} .
\end{aligned}
$$

В более общем, неизотропном случае МЭ определяются формулой, аналогичной (4), однако в качестве базисных функций вместо полиномов Сонина используются функции Барнетта $H_{r l m}=S_{l+1 / 2}^{p}(x) Y_{l, m}(\theta, \phi)$.

Как показано в $[4,8]$, изотропные МЭ $\left(K_{a, b}^{a}\right)$ с различными индексами связаны соотношением

$$
\begin{aligned}
& T \frac{d\left(K_{a, b}^{a}\right)_{r_{1}, r_{2}}^{r}(T)}{d T}-\left(r_{1}+r_{2}-r\right)\left(K_{a, b}^{a}\right)_{r_{1}, r_{2}}^{r}(T) \\
= & r\left(K_{a, b}^{a}\right)_{r_{1}, r_{2}}^{r-1}(T)-\left(r_{1}+1\right)\left(K_{a, b}^{a}\right)_{r_{1}+1, r_{2}}^{r}(T) \\
& -\left(r_{2}+1\right)\left(K_{a, b}^{a}\right)_{r_{1}, r_{2}+1}^{r}(T) .
\end{aligned}
$$

В случае степенной зависимости потенциала $\varphi$ от расстояния $r\left(\varphi \sim 1 / r^{\kappa}\right)$ сечение рассеяния представляется в виде

$$
\sigma_{a b}(g, \theta)=g^{\gamma-1} F_{\gamma}^{a b}(z) .
$$

Здесь $z=\sin ^{2}(\theta / 2), \gamma=(\kappa-4) / \kappa, F_{\gamma}^{a b}(\theta)-$ угловая часть сечения, $\theta-$ угол рассеяния.

В случае сечения рассеяния (7) МЭ пропорционален $T^{\mu}$, где $\mu=\gamma / 2$ (см., например, [9]). Соотношения (6) становятся алгебраическими и представляют собой рекуррентные соотношения:

$$
\begin{aligned}
& \left(\mu-r_{1}-r_{2}+r\right)\left(K_{a, b}^{a}\right)_{r_{1}, r_{2}}^{r}(T)=r\left(K_{a, b}^{a}\right)_{r_{1}, r_{2}}^{r-1}(T) \\
& -\left(r_{1}+1\right)\left(K_{a, b}^{a}\right)_{r_{1}+1, r_{2}}^{r}(T)-\left(r_{2}+1\right)\left(K_{a, b}^{a}\right)_{r_{1}, r_{2}+1}^{r}(T) .
\end{aligned}
$$

Существование рекуррентных соотношений позволяет построить рекуррентную процедуру, в которой стартовыми являются линейные МЭ $K_{r_{1}, 0}^{r}$ (первый тип) или $K_{0, r_{2}}^{r}$ (второй тип). В случае простого газа для этих стартовых МЭ получены простые аналитические формулы $[4,8]$. На основе рекуррентных соотношений (8) нами была реализована программа расчета МЭ с большими индексами (с индексами порядка сотни и более), для чего оказалось необходимым разработать специальные процедуры точной арифметики. Это позволило в изотропном случае решать нелинейное уравнение Больцмана и рассчитывать функцию распределения вплоть до 8-10 тепловых скоростей. Для максвелловских молекул расчет проводился до 12 тепловых скоростей. Было проведено сравнение с аналитическим решением БКВ $[\mathrm{BKW}]$ и получено полное совпадение. При этом рассматривалось отношение $Ф Р$ к равновесному максвеллиану.

В случае произвольных законов взаимодействия в $[1,2]$ аналитически проведен расчет линейных неизотропных МЭ при малых значениях индекса $l(l=1,2)$, отвечающего за разложения ФР по сферическим гармоникам. В результате такие МЭ представляются в виде линейной комбинации двойных интегралов ( $\Omega$-интегралов), содержащих сечение взаимодействия $\sigma_{a b}(g, \theta)$.

Следуя [2], запишем $\Omega$-интегралы в виде

$$
\Omega_{a b}^{(n, m)}=\left(\frac{k T}{2 \pi m_{a b}}\right)^{1 / 2} \int_{0}^{\infty} e^{-\bar{g}^{2}} \bar{g}^{2 m+3} Q_{a b}^{(n)} d \bar{g},
$$

где $m_{a b}=\left(m_{a} m_{b}\right) /\left(m_{a}+m_{b}\right)$ - приведенная масса пары молекул сортов $a$ и $b$, а безразмерная переменная интегрирования $\bar{g}=g \sqrt{m_{a b} / 2 k T}$. Величина $Q_{a b}^{(n)}$ в (9) определяется выражением

$$
Q_{a b}^{(n)}=2 \pi \int_{0}^{\pi}\left(1-\cos ^{n} \theta\right) \sigma_{a b}(g, \theta) \sin \theta d \theta .
$$

В [11] по аналогии с хорошо известным из кинетической теории методом расчета интегральных скобок $[1,2]$ полу- 
чены аналитические представления изотропных линейных МЭ как первого, так и второго типов в виде конечных сумм $\Omega$-интегралов. При этом так же, как и в $[1,2]$, использована производящая функция для полиномов Сонина, выбираются те же переменные интегрирования, так же проводится изменение порядков многочисленных суммирований. В изотропном случае расчеты оказались значительно более простыми. Особенно просто через $\Omega$-интегралы выражаются линейные изотропные МЭ второго типа $K_{0, r_{2}}^{r}$, поэтому имеет смысл выбирать коэффициенты разложения именно этих МЭ в качестве стартовых в рекуррентной процедуре получения всех изотропных МЭ. При этом удобно вместо функций $Q_{a b}^{(n)}$ использовать

$$
Q_{* a b}^{(n)}=2 \pi\left(1-\delta_{n, 0}\right) \int_{0}^{\pi}(1-\cos \theta)^{n} \sigma_{a b}(g, \theta) \sin \theta d \theta .
$$

По аналогии с $\Omega$-интегралами определим $\Omega_{*}$-интегралы

$$
\Omega_{* a b}^{(n, m)}=\left(\frac{k T}{2 \pi m_{a b}}\right)^{1 / 2} \int_{0}^{\infty} e^{-\bar{g}^{2}} \bar{g}^{2 m+3} Q_{* a b}^{(n)} d \bar{g} .
$$

$\Omega_{*}$-интегралы легко выражаются через $\Omega$-интегралы, и наоборот:

$$
\begin{aligned}
& \Omega_{* a b}^{(n, m)}=\sum_{l=0}^{n}\left(\begin{array}{l}
n \\
l
\end{array}\right)(-1)^{l+1} \Omega_{a b}^{(l, m)}, \\
& \Omega_{a b}^{(n, m)}=\sum_{l=1}^{n}\left(\begin{array}{l}
n \\
l
\end{array}\right)(-1)^{l+1} \Omega_{* a b}^{(l, m)} .
\end{aligned}
$$

Приведем здесь полученные в [11] представления линейных изотропных МЭ первого и второго типов через $\Omega_{*}$ интегралы. Для МЭ первого типа имеем

$$
\begin{gathered}
K_{p, 0}^{q}=(-1)^{p+q} \sum_{n=1}^{\min (p, q)} \sum_{m=n}^{p+q-n} A_{p, q ; n, m}^{*(1)} \Omega_{* a b}^{(n, m)} \\
A_{p, q ; n, m}^{*(1)}=8 v_{q}^{-1} \mu_{a}^{n} \mu_{b}^{m} \sum_{j=j_{0}}^{m-n} \frac{2^{m-j}}{n ! j !(m-j-n) !} \sum_{i=i_{0}}^{s-2 m} G_{p q j i m} \\
G_{p q j i m}=\frac{(-1)^{i+n} \mu_{b}^{i}\left(1-\mu_{a} / \mu_{b}\right)^{s-i-2 m}}{(i+m-p) !(i+m-q) !(s-i-2 m) !} \\
\times \frac{\Gamma(i+m+3 / 2)(2(i+m)+j-s) !}{\Gamma(m+3 / 2)(2(i+m)-s) !}, s=j+p+q .
\end{gathered}
$$

Здесь

$$
\begin{gathered}
j_{0}=\max (0, m-p, m-q, 2 m-p-q), \\
i_{0}=\max (0, p-m, q-m,(j+p+q-2 m) / 2) .
\end{gathered}
$$

Матричные элементы второго типа выражаются через $\Omega_{*}$-интегралы следующим образом:

$$
\left(K_{a, b}^{a}\right)_{0, p}^{q}=-8 v_{q}^{-1} \mu_{b}^{p} \mu_{a}^{q} \sum_{n=1}^{\min (p, q)} \sum_{m=n}^{p+q-n} A_{p, q ; n, m}^{*} \Omega_{* a b}^{(n, m)},
$$

где

$$
\begin{aligned}
A_{p, q ; n, m}^{*(2)}= & \frac{2^{n} \Gamma(p+q-n+3 / 2)}{n !(p-n) !(q-n) !} \\
& \times\left(\begin{array}{c}
p+q-2 n \\
m-n
\end{array}\right) \frac{(-1)^{m+n}}{\Gamma(m+3 / 2)} .
\end{aligned}
$$

Из этих формул понятно, что представление МЭ второго типа через $\Omega_{*}$-интегралы значительно проще. Поэтому естественно стартовать в рекуррентной процедуре с МЭ второго типа.

\section{Построение нелинейных матричных элементов}

Покажем, что, стартуя с линейных МЭ второго типа (16) с помощью соотношений (6), можно построить все изотропные МЭ как нелинейные, так и линейные первого типа. Несмотря на то что в (6) входит производная по температуре, связи между коэффициентами разложения МЭ по $\Omega$-интегралам, как будет показано ниже, оказываются чисто алгебраическими.

Учитывая (13), легко убедиться, что $\Omega_{*}$-интегралы связаны между собой так же, как и $\Omega$-интегралы ([2]):

$$
T \frac{\partial \Omega_{* a b}^{(n, m)}}{\partial T}+(m+3 / 2) \Omega_{* a b}^{(n, m)}=\Omega_{* a b}^{(n, m+1)} .
$$

Запишем рекуррентное соотношение (6) в виде

$$
\begin{aligned}
& \left(r_{1}+1\right)\left(K_{a, b}^{a}\right)_{r_{1}+1, r_{2}}^{r}=-\left(r_{2}+1\right)\left(K_{a, b}^{a}\right)_{r_{1}, r_{2}+1}^{r} \\
& -T \frac{d\left(K_{a, b}^{a}\right)_{r_{1}, r_{2}}^{r}}{d T}+\left(r_{1}+r_{2}-r\right)\left(K_{a, b}^{a}\right)_{r_{1}, r_{2}}^{r}+r\left(K_{a, b}^{a}\right)_{r_{1}, r_{2}}^{r-1} .
\end{aligned}
$$

В дальнейшем удобно в качестве второго нижнего индекса в матричном элементе $\left(K_{a, b}^{a}\right)_{r_{1}, r_{2}}^{r}$ использовать не $r_{2}$, а $N=r_{1}+r_{2}$. Обозначим соответствующий МЭ через $K_{r_{1}, N}^{\prime r}$, т. е.

$$
K_{r_{1}, N}^{\prime r}=\left(K_{a, b}^{a}\right)_{r_{1}, N-r_{1}}^{r}
$$

В этих обозначениях после замены $N$ на $N-1$ (19) принимает вид

$$
\begin{aligned}
& \left(r_{1}+1\right) K_{r_{1}+1, N}^{\prime r}=-\left(N-r_{1}\right) K_{r_{1}, N}^{\prime r}-T \frac{d}{d T} K_{r_{1}, N-1}^{\prime r} \\
& +(N-1-r) K_{r_{1}, N-1}^{\prime r}+r K_{r_{1}, N-1}^{r_{1}-1} .
\end{aligned}
$$

При $r_{1}=0$ в соответствии с (16) и (20) запишем матрицу $K^{\prime}$ в виде

$$
K_{0, N}^{\prime r}=\sum_{n=1}^{\min (N, r)} \sum_{m=n}^{N+r-n} \beta_{0, N}^{r}(n, m) \Omega_{* a b}^{(n, m)}
$$

где

$$
\beta_{0, N}^{r}(n, m)=8 v_{r}^{-1} \mu_{b}^{N} \mu_{a}^{r} A_{N, r ; n, m}^{*}
$$


Из (22) видно, что $K_{0, N}^{\prime r}$ представляет собой линейную комбинацию $\Omega_{*}$-интегралов, где $n$ и $m$ изменяются в областях

$$
1 \leq n \leq \min (N, r), \quad n \leq m \leq N+r-n .
$$

Рассмотрим рекуррентное соотношение (21) при $r_{1}=0$. Сначала, используя (18), перепишем член, связанный с производной:

$$
\begin{aligned}
& T \frac{d}{d T} K_{0, N-1}^{\prime r}=\sum_{n=1}^{\min (N-1, r)} \sum_{m=n+1}^{N+r-n} \beta_{0, N-1}^{r}(n, m) \Omega_{* a b}^{(n, m+1)} \\
& -\sum_{n=1}^{\min (N-1, r)} \sum_{m=n}^{N-1+r-n}(m+3 / 2) \beta_{0, N}^{r}(n, m) \Omega_{* a b}^{(n, m)}
\end{aligned}
$$

Переходя в первой из двойных сумм в (25) от переменной суммирования $m$ к $m-1$ и подставляя результат в (21), получаем

$$
\begin{aligned}
& K_{1, N}^{\prime r}=-N K_{0, N}^{\prime r}+(N-1-r) K_{0, N-1}^{\prime r}+r K_{0, N-1}^{\prime r-1} \\
& -\sum_{n=1}^{\min (N-1, r)} \sum_{m=n+1}^{N+r-n} \beta_{0, N-1}^{r}(n, m-1) \Omega_{* a b}^{(n, m)} \\
& +\sum_{n=1}^{\min (N-1, r)} \sum_{m=n}^{N-1+r-n}(m+3 / 2) \beta_{0, N-1}^{r}(n, m) \Omega_{* a b}^{(n, m)} .
\end{aligned}
$$

Отсюда видно, что пределы суммирования по $n, m$ не расширяются при дифференцировании $K_{0, N}^{\prime r}$ по температуре. Подставляя в (26) МЭ в виде (22), получим разложение $K_{1, N}^{\prime r}$ по $\Omega_{*}$-интегралам, которое имеет такую же структуру, как (22). Отметим, что область суммирования (24) при этом не изменяется. Последовательно увеличивая $r_{1}$, можно таким же образом показать, что и в общем случае пределы суммирования в $K_{r_{1}+1, N}^{\prime r}$ не зависят от $r_{1}$, и произвольный матричный элемент представляется в виде линейной комбинации

$$
K_{r_{1}, N}^{\prime r}=\sum_{n=1}^{\min (N, r)} \sum_{m=n}^{N+r-n} \beta_{r_{1}, N}^{r}(n, m) \Omega_{* a b}^{(n, m)} .
$$

Понятно, что такая же формула с другими коэффицинтами справедлива и при разложении по $\Omega$-интегралам.

Для $n>\min (N, r)$ и $m>N+r-n$ доопределим матрицу $\beta_{r_{1}, N}^{r}(n, m)$ нулями. Тогда можно записать

$$
K_{r_{1}, N}^{\prime r}=\sum_{n=1}^{\infty} \sum_{m=n}^{\infty} \beta_{r_{1}, N}^{r}(n, m) \Omega_{* a b}^{(n, m)} .
$$

Подставляя последнее равенство в (21) и приравнивая коэффициенты при одинаковых $\Omega_{*}$-интегралах, получим следующее рекуррентное соотношение, где с помощью
$\Theta$-функций явно выделены области ненулевых значений $\beta_{r_{1}, N}^{r}(n, m)$ :

$$
\begin{aligned}
& \beta_{r_{1}+1, N}^{r}(n, m)=\frac{1}{r_{1}+1}\left[-\left(N-r_{1}\right)\right. \\
& \times \Theta(\min (N, r)-n) \Theta(N+r-n-m) \beta_{r_{1}, N}^{r}(n, m) \\
& +(m+N-1-r+3 / 2) \Theta(\min (N-1, r)-n) \\
& \times \Theta(N-1+r-n-m) \beta_{r_{1}, N-1}^{r}(n, m) \\
& +r \Theta(\min (N-1, r-1)-n) \\
& \times \Theta(N+r-2-n-m) \beta_{r_{1}, N-1}^{r-1}(n, m)- \\
& \times \Theta(\min (N-1, r)-n) \Theta(N+r-n-m) \\
& \left.\times\left(1-\delta_{m, n}\right) \beta_{r_{1}, N-1}^{r}(n, m-1)\right] .
\end{aligned}
$$

Эта же рекуррентная формула справедлива и при разложении по $\Omega$-интегралам. Различие будет только в начальных коэффициентах разложения $\beta_{0, N}^{r}(n, m)$. Особо отметим универсальность рекуррентной формулы (28) для матрицы $\beta$. При любом фиксированном отношении масс она не зависит от сечения взаимодействия.

Нами была составлена программа расчета матрицы $\beta$ по формуле (28). Получено полное совпадение коэффициентов разложения линейных изотропных МЭ первого типа $\left(K_{a, b}^{a}\right)_{0, p}^{q}$ по $\Omega$-интегралам с аналитически найденными в [12] при нескольких первых значениях индексов. Подчеркнем, что в нашем подходе такие МЭ вычисляются в самом конце рекуррентной процедуры после определения всех нелинейных МЭ.

Таким образом, показано, что связи между МЭ могут быть использованы как рекуррентные соотношения при вычислении нелинейных МЭ для произвольных законов взаимодействия.

Отметим, что расчет коэффициентов разложения $\beta_{r_{1}, N}^{r}(n, m)$ должен проводиться с высокой точностью. Для реализации расчета с учетом большого количества значащих цифр мы использовали пакет MPFUN2015 [13]. Было проведено сравнение изотропных нелинейных МЭ $K_{r_{1}, r_{2}}^{r}$ (15 значащих цифр) с индексами в диапазоне $[0,32]$, рассчитанных с помощью формул $(27),(28)$ и найденных с помощью рекуррентной формулы (8) для случая твердых шаров. Получено полное совпадение результатов.

\section{Степенные законы взаимодействия}

Найдем выражение для $\Omega_{* a b}^{(n, m)}$ в случае степенных законов взаимодействия. Подставляя сечение (7) в выражение для $Q_{* a b}^{(n)}(11)$, после интегрирования по $\bar{g}$ 
получаем

$$
\Omega_{* a b}^{(n, m)}=2^{\mu-2} \pi^{-1 / 2}\left(\frac{k T}{m_{a b}}\right)^{\mu} 2^{n} J_{n}(\mu) \Gamma(m+3 / 2+\mu),
$$

где величина $J_{n}(\mu)$ в отличие от случая произвольных потенциалов связана только с угловой частью сечения:

$$
J_{n}(\mu)=4 \pi \int_{0}^{1} F_{\gamma}^{a b}(z) z^{n} d z
$$

Покажем, что в этом случае выражение для линейных изотропных МЭ второго рода, которые являются стартовыми в рекуррентной процедуре, основанной на (8), оказываются существенно проще, чем в общем случае. Для этого подставим представление $\Omega_{* a b}(29)$ в разложение (16), (17):

$$
\begin{aligned}
& K_{0, p}^{q}=-\frac{2^{\mu+1}}{\pi^{1 / 2} v_{q}}\left(\frac{k T}{m_{a b}}\right)^{\mu} \mu_{b}^{p} \mu_{a}^{q} \\
& \times \sum_{n=1}^{\min (p, q)} J_{n}(\mu) \frac{2^{2 n} \Gamma(p+q-n+3 / 2)}{n !(p-n) !(q-n) !} \\
& \times \sum_{s=0}^{p+q-2 n}\left(\begin{array}{c}
p+q-2 n \\
s
\end{array}\right)(-1)^{s} \frac{\Gamma(n+s+3 / 2+\mu)}{\Gamma(n+s+3 / 2)} .
\end{aligned}
$$

Используя интегральное представление $B$-функции [14], можно показать, что

$$
\begin{aligned}
\sum_{k=0}^{t}\left(\begin{array}{l}
t \\
k
\end{array}\right)(-1)^{k} \frac{\Gamma(a+k)}{\Gamma(b+k)} & =\frac{\Gamma(a) \Gamma(t+b-a)}{\Gamma(b-a) \Gamma(t-b)} \\
a>0, \quad b & >0 .
\end{aligned}
$$

Заменяя последнюю сумму в (31) выражением (32) при $t=p+q-2 n, a=n+3 / 2+\mu$, и $b=n+3 / 2$, получаем представление линейных изотропных МЭ второго типа в виде однократной суммы

$$
\begin{aligned}
& K_{0, p}^{q}=\left(\frac{2 k T}{m_{a b}}\right)^{\mu} \mu_{b}^{p} \mu_{a}^{q} \frac{q !}{\Gamma(q+3 / 2)} \sum_{n=1}^{\min (p, q)} \\
& \times J_{n} \frac{2^{2 n} \Gamma(n+3 / 2+\mu) \Gamma(p+q-2 n-\mu)}{n !(p-n) !(q-n) ! \Gamma(-\mu)} .
\end{aligned}
$$

Таким образом, в случае степенных законов взаимодействия получена простая формула для линейных изотропных МЭ второго типа с произвольным соотношением масс. Эта формула для простого газа $\left(\mu_{a}=\mu_{b}=1 / 2\right.$, $\left.m_{a b}=m / 2\right)$ совпадает с выражением, полученным ранее другим способом в $[4,8]$.

Выразим $J_{n}$ через параметры степенного потенциала. Из (10) следует, что для степенных потенциалов

$$
Q_{* a b}^{(n)}=g^{2 \mu-1} 2^{n} J_{n}(\mu) .
$$

Рассмотрим потенциал

$$
\varphi=\left(\frac{\epsilon}{r}\right)^{\nu}, \quad v=\frac{4}{1-2 \mu}
$$

Известно (см., например, [2]), что в случае потенциала (35) $Q_{a b}^{(n)}(10)$ можно представить в виде

$$
Q_{a b}^{(n)}=2 \pi \epsilon^{2}\left(\frac{m_{a b} g^{2}}{2 v}\right)^{-2 / v} A_{n}(v)
$$

где

$$
A_{n}(v)=\int_{0}^{\infty}\left(1-\cos ^{n}(\theta(s, v))\right) s d s
$$

Способ построения угла $\theta(s, v)$ можно найти, например, в [2]. Там же приведены значения $A_{n}(v)$ при $n=1,2$ для ряда целых значений $v$ из области $4 \leq v \leq \infty$.

Аналогичная формула связывает $Q_{* a b}^{(n)}$ и величину

$$
\tilde{A}_{n}(v)=\int_{0}^{\infty}(1-\cos (\theta(s, v)))^{n} s d s .
$$

Из (34), представляя $Q_{* a b}^{(n)}$ в виде, аналогичном (36), получаем

$$
J_{n}(\mu)=\pi \epsilon^{2}\left(\frac{m_{a b}}{2 v}\right)^{-2 / v} \tilde{A}_{n}(v) .
$$

Таким величина $J_{n}(\mu)$ под знаком суммы в (33) полностью определена.

\section{Заключение}

При расчете сильно неравновесных ФР моментным методом необходимо учитывать большое число членов в разложении ФР, следовательно, необходимы матричные элементы с большими индексами. В наших работах $[4,8]$ показано, что МЭ с соседними индексами связаны соотношениями, которые следуют из инвариантности интеграла столкновений относительно выбора параметров весового максвеллиана в разложении по функциям Барнетта, а именно температуры и средней скорости.

В случае степенных законов взаимодействия все соотношения, связывающие МЭ, являются алгебраическими. Это позволило построить рекуррентную процедуру для последовательного определения МЭ $[4,8]$. Найденные МЭ были использованы для расчета ФР при сильном отклонении от равновесия в частности, в сильных постоянных и переменных внешних полях [15].

Для произвольных законов взаимодействия соотношения, связывающие неизотропные МЭ (они следуют из инвариантности относительно выбора средней скорости весового максвеллиана), также являются алгебраическими. На их основе в [6] была построена рекуррентная процедура, которая позволяет найти все неизотропные 
МЭ, если известны изотропные МЭ. Таким образом, построение изотропных МЭ приобретает особое значение.

Для произвольных (не степенных) законов взаимодействия соотношения, связывающие изотропные МЭ, включают производную по температуре. Переходу к алгебраическим соотношениям помогает использование хорошо известных в кинетической теории $\Omega$ интегралов. Учитывая, что производная от $\Omega$-интеграла по температуре выражается через линейную комбинацию $\Omega$-интегралов, удается записать алгебраические соотношения для коэффициентов разложения МЭ по $\Omega$-интегралам. При этом оказалось, что более удобно рассматривать коэффициенты разложения по $\Omega_{*}$ интегралам (12), которые легко выражаются через $\Omega$ интегралы. Эти коэффициенты последовательно определяются с помощью описанной в настоящей работе рекуррентной процедуры, причем в качестве стартовых целесообразно использовать коэффициенты разложения линейных изотропных МЭ второго типа $K_{0, r_{2}}^{r}$, которые имеют наиболее простой вид (17). Необходимо отметить, что соотношения, связывающие коэффициенты разложения, являются общими для $\Omega_{*}$ - и $\Omega$-интегралов, они также не зависят от масс сталкивающихся частиц. Отношение масс входит только в стартовые коэффициенты разложения (17).

Нами была реализована процедура, позволяющая последовательно вычислять все изотропные МЭ с использованием в качестве входных данных набора известных (табулированных) $\Omega$-интегралов. Она требует вычислений с повышенной точностью, для чего был использован пакет MPFUN2015 [13]. Программа была протестирована на примере вычисления МЭ для модели твердых шаров.

Для степенных законов взаимодействия представление линейных изотропных МЭ второго типа в виде разложения по $\Omega_{*}$-интегралам позволило обобщить полученную в $[4,8]$ формулу на случай произвольного соотношения масс. Их разложение при этом сводится к однократной сумме.

Для произвольных законов взаимодействия изотропные нелинейные МЭ могут быть рассчитаны по формуле (27), в которой коэффициенты $\beta_{r_{1}+1, N}^{r}(n, m)$ определяются с помощью рекуррентной процедуры. В свою очередь, как было указано выше, они являются стартовыми для расчета неизотропных нелинейных МЭ. Таким образом, совместное применение описанных в этой работе и в нашей предыдущей работе [6] рекуррентных процедур дает возможность найти все МЭ нелинейного интеграла столкновений для произвольных законов взаимодействия и произвольных масс сталкивающихся частиц. Табулированные МЭ могут затем использоваться для решения различных задач. Это существенно расширяет возможности моментного метода, который может теперь применяться для расчета сильно неравновесных ФР в реальных физических ситуациях.

\section{Список литературы}

[1] Чепмен С., Каулинг Т. Математическая теория неоднородных газов. М.: Изд-во иностр. лит-ры, 1960. 510 с.

[2] Ферцигер Дж., Капер Г. Математическая теория процессов переноса в газах. М.: Мир, 1976. 557 с.

[3] Viehland L.A., Maeson E.A. // Ann. Phys. 1978. Vol. 110. P. $287-328$

[4] Эндер А.Я., Эндер И.А. Интеграл столкновений уравнения Больцмана и моментный метод. СПб., 2003. 224 с.

[5] Ender A.Ya., Ender I.A. // Transport Theor. Stat. Phys. 2007. Vol. 56. P. 563-588.

[6] Эндер И.А., Бакалейников Л.А., Флегонтова Е.Ю., Герасименко А.Б. // ЖТФ. 2017. Т. 87. № 8.

[7] Shizgal B. D., Dridi R. // Computer Phys. Commun. 2010. Vol. 181. P. 1633-1640.

[8] Ender A.Ya., Ender I.A. // Phys. Fluids. 1999. Vol. 11. P. 2720-2730.

[9] Эндер А.Я., Эндер И.А. // ЖТФ. 1994. Т. 64. Вып. 10. С. 38 53.

[10] Бобылев А.В. // Докл. АН СССР. 1975. Т. 225. Вып. 5. C. 1041

[11] Эндер А.Я., Эндер И.А., Лютенко М.Б. Полиномиальное разложение изотропного уравнения Больцмана и независимость интеграла столкновений относительно выбора бвзисных функций. Препринт ФТИ РАН № 1716. СПб., 1998. $62 \mathrm{c}$.

[12] Мак-Даниэль И., Мэзон Д. Подвижность и диффузия ионов в газах. М.: Мир, 1976. 422 с.

[13] David H. Bailey MPFUN2015: A thread-safe arbitrary precision package. http://www.davidhbailey.com/dhbsoftware/

[14] Градштейн И.С., Рыжик И.М. Таблицы интегралов, сумм, рядов и произведений. М.: ФМ., 1962. 1097 с.

[15] Ender A.Y., Ender I.A., Gerasimenko A.B. // Open Plasma Phys. J. 2009. Vol. 2. P. 24-62. 